\title{
Mobility of the gradient tracking machine in mating yeast depends on Bud1 inactivation and actin- independent vesicle delivery
}

\section{Xin Wang}

University of Illinois at Chicago https://orcid.org/0000-0002-3891-2684

David Stone ( $\nabla$ dstone@uic.edu )

University of Illinois at Chicago

\section{Article}

Keywords: Bud1 inactivation, actin-independent vesicle delivery, gradient tracking machine (GTM)

Posted Date: June 3rd, 2021

DOI: https://doi.org/10.21203/rs.3.rs-567980/v1

License: (c) (1) This work is licensed under a Creative Commons Attribution 4.0 International License.

Read Full License 


\title{
Mobility of the gradient tracking machine in mating yeast depends on Bud1 inactivation and actin-independent vesicle delivery
}

Running title: Regulation of polarity-site function and vesicle delivery enables gradient tracking

Xin Wang ${ }^{1}$ and David E. Stone ${ }^{1 *}$

\author{
Affiliations: \\ ${ }^{1}$ Department of Biological Sciences, University of Illinois at Chicago, Chicago, IL 60607,
} USA.

*Correspondence to: D. S. at the Department of Biological Sciences, University of Illinois at Chicago, 900 South Ashland Avenue, Chicago, IL 60607, USA; e-mail: dstone@uic.edu; phone: 312-996-5710; fax: 312-413-2691. 
ABSTRACT: The mating of budding yeast depends on chemotropism, a fundamental cellular process. Haploid yeast cells of opposite mating type signal their positions to one another through the secretion of mating pheromones. We have proposed a deterministic gradient sensing model that explains how these cells orient toward their mating partners. Using the cell-cycle determined default polarity site (DS), cells assemble a gradient tracking machine (GTM) composed of signaling, polarity, and trafficking proteins. After assembly, the GTM redistributes up the gradient, aligns with the pheromone source, and triggers polarized growth toward the partner. Because strong positive feedback mechanisms drive polarized growth at the DS, it is unclear how the GTM is released for tracking after its assembly is complete. What prevents the GTM from triggering polarized growth at the DS? Here we describe two mechanisms that enable tracking. First, the Ras GTPase Bud1 must be inactivated to release the GTM. Second, actinindependent - but not actin-dependent - vesicle delivery must be targeted upgradient to effect GTM redistribution. 


\section{INTRODUCTION}

Cellular responses to chemical gradients are important for a wide range of biological phenomena. The best-known gradient-stimulated outputs are chemotaxis (directed movement) and chemotropism (directed growth). For example, chemotaxis plays a vital role in development, immunity, wound healing, inflammation, and $\operatorname{metastasis}^{1}$; chemotropism is integral to axon guidance, angiogenesis, pollen tube guidance, and fungal life cycles ${ }^{2,3}$. Although they exhibit different behaviors, chemotactic and chemotropic cells face similar challenges: the responding cell must determine the direction of the gradient source by sensing small chemical concentration differences across its surface, then correctly polarize its cytoskeleton.

The unicellular eukaryote, Saccharomyces cerevisiae, is among the best-studied models of both cell-cycle control and chemotropism ${ }^{4}$. During vegetative growth, haploid yeast cells invariably form new buds adjacent to their last division site, resulting in a characteristic axial budding pattern ${ }^{5}$. Late in the G1 phase of the cell cycle, the Ax12 cortical marker protein recruits the Bud5 guanine nucleotide exchange factor (GEF) to the axial bud site, where it activates its target, the Ras GTPase Bud1 ${ }^{6,7}$. Activated Bud1 interacts with the Rho GTPase Cdc42 and its GEF Cdc24, initiating local activation of $\mathrm{Cdc} 42^{8}$. This positional signal is greatly amplified by two positive feedback loops and the resulting concentrated patch of active Cdc42 triggers the nucleation of actin cables and polarized delivery of secretory vesicles ${ }^{9-12}$. During the sexual reproduction stage of their lifecycle, haploid yeast cells differentiate into gametes and fuse to form diploid zygotes. Each of the two haploid mating types, MATa and MATa, secretes a peptide pheromone that activates a G-protein-coupled receptor (GPCR) on cells of the opposite type. The pheromone-bound receptor activates its cognate heterotrimeric G protein, causing G $\alpha$-GTP to dissociate from G $\beta \gamma$. Free G $\beta \gamma$ then signals to the nucleus through the Fus3 MAPK cascade, 
inducing changes in gene expression and cell-cycle arrest in late G1. G $\beta \gamma$ also positions the eventual chemotropic growth site (CS) by linking the receptor to the machinery that nucleates actin cables via the Far1 scaffold protein ${ }^{13-15}$. Actin-directed delivery of secretory vesicles to the CS results in the formation of mating projections, commonly known as "shmoos." In mating mixtures, cells find and contact a partner by determining the direction of the most potent pheromone source and polarizing their growth toward it (hereafter referred to as shmooing) ${ }^{16}$. When cells are treated with isotropic pheromone or are unable to sense a gradient, however, they shmoo adjacent to their last bud site - i.e., at the axial site where they would have budded next if not arrested in $\mathrm{G} 1^{17,18}$. Hence, the axial bud site is also referred to as the default polarity site $(\mathrm{DS})^{14,18}$.

Like all other chemo-sensing cells, yeast exhibit a remarkable ability to interpret chemoattractant gradients. It has been estimated that a $1 \%$ difference in receptor activation across the $5 \mu \mathrm{m}$ diameter of a yeast cell is sufficient to elicit robust orientation ${ }^{19}$. In mating mixtures, yeast cells almost invariably select a single partner, even when surrounded by multiple potential mates. We have recently proposed a deterministic gradient sensing model that explains how mating yeast cells accurately position the CS in response to shallow and dynamic physiological gradients ${ }^{20,21}$. In this model, yeast cells gain their gradient-sensing ability and orient toward their mating partners in four phases. During global internalization, the uniformly distributed receptor and G protein are removed from the plasma membrane (PM). During assembly, mating yeast cells take advantage of the Bud1-positioned DS to assemble the signaling, polarity, and trafficking proteins into a gradient tracking machine (GTM). Assembly of the GTM starts with Far1-Cdc24-Bem1 localization to the DS and ends with the concentration of exocytic and endocytic activities upgradient and downgradient, respectively. During tracking, 
segregation of exocytosis and endocytosis incrementally redistributes the GTM up the gradient along the PM to the CS. At the CS, the GTM stabilizes when vesicle delivery aligns with the pheromone gradient and the endocytic machinery surrounds the secretion site.

Although our model explains how yeast cells actively track pheromone gradients, it leaves a key question unanswered: How does tracking start? That is, how is the newly assembled GTM released from the DS? The challenge is to understand how the subtle directional information embedded in the extracellular pheromone gradient overrides the strong, feedback-amplified polarity of this intrinsic site. Here, we provide evidence for two mechanisms that explain how the GTM "escapes" from the DS and begins tracking. First, we show that the Bud1 GEF disappears from the PM in cells preparing to mate, whereas the Bud1 GTPase-activating protein (GAP), Bud2, polarizes to the DS and tracks with the receptor. We also demonstrate that tracking requires Bud1 inactivation. Second, we show that the mode of vesicle delivery changes as the GTM transitions from assembly to tracking and from tracking to stabilization. Whereas actindependent vesicle delivery (AD-VD) is active when the GTM is immobile, both before and after tracking, actin-independent vesicle delivery (AI-VD) is necessary and sufficient during tracking. Our findings suggest that tight regulation of both DS function and the modes of vesicle delivery is essential for yeast gradient sensing. 


\section{RESULTS}

\section{Bud5-RFP fails to localize to the DS and disappears from the PM in mating cells, whereas}

RFP-Bud2 polarizes to the DS and tracks with the receptor

During the G1 phase of vegetative haploid cells, the Bud1 GTPase is activated adjacent to the cytokinesis site, where it marks the DS as the bud site in the next cell cycle. Active Bud1 also positions shmoo formation at the DS in cells treated with isotropic pheromone. A longstanding question in the study of yeast mating is how shallow pheromone gradients compete with the polarization machinery at a cell's DS to establish a CS aligned with that of its partner. We have shown that G1-arrested yeast cells preparing to mate assemble a GTM composed of signaling, polarity, and trafficking proteins at the DS, which enables them to find the closest mating partner $^{20}$. Moreover, the positioning of the GTM at the DS during mating, like the positioning of the daughter cell during vegetative growth, depends on Bud1: in the absence of Bud1, mating cells assemble multiple GTMs at random positions on the PM. These observations led us to hypothesize that the release of the GTM from the DS to start tracking requires the inactivation of Bud1.

To test this idea, we engineered MATa cells co-expressing the receptor reporter (Ste2-GFP) as a proxy for the GTM, and either RFP-tagged Bud5, the Bud1 GEF ${ }^{7}$, or RFP-tagged Bud2, the Bud1 GAP ${ }^{22}$. We took time-lapse images of these MATa cells during vegetative growth and in mating mixtures. In vegetative cells, Bud5-RFP localized to the DS after cytokinesis and before bud emergence (Fig. 1A), whereas RFP-Bud2 concentrated at the bud neck but was not detectable at the PM between cytokinesis and bud emergence (Fig. 1B). These observations are consistent with those reported by Park et al. ${ }^{22}$ and Marston et al. ${ }^{7}$ Conversely, in mating yeast, Bud5-RFP gradually disappeared from the mother-daughter neck and was never detectable at the 
DS or elsewhere on the PM (Fig. 1C), whereas RFP-Bud2 polarized to the DS after cytokinesis and before the receptor (Fig. 1D). The polarized RFP-Bud2 and receptor tracked together along the PM to the CS before shmoo formation and fusion. These data suggest that Bud1 is inactivated in mating cells before tracking begins.

\section{Tracking is defective in mating cells expressing Bud1 ${ }^{\mathrm{G} 12 \mathrm{~V}}$}

The absence of the Bud1 GEF (Bud5) from the DS along with the localization of the Bud1 GAP (Bud2) to the GTM during both assembly at the DS and redistribution suggested to us that Bud1 inactivation is required for gradient tracking. To test this, we imaged the receptor reporter in mating MATa $B U D 1$ cells expressing a constitutively active form of Bud1, Bud1 ${ }^{\mathrm{G} 12 \mathrm{~V}}$, from a centromeric plasmid (hereafter, $B U D 1^{G 12 V} / B U D 1$ cells) (Fig. 2$)^{23}$. The G12V amino acid substitution blocks the GTPase activity of Bud1, thereby locking it in the active state. Cells forced to express Bud1 ${ }^{\mathrm{G} 12 \mathrm{~V}}$ in the absence of Bud1 are not viable. Consistent with our hypothesis, about $30 \%$ of the $B U D 1^{G 12 V} / B U D 1$ cells ignored $M A T \alpha$ cells with which they were in direct contact, a behavior we see one tenth as often in wild-type (WT) mating mixtures. These $B U D 1^{G 12 V} / B U D 1$ cells either continued to bud (Fig. $2 \mathrm{~A}$ ) or failed to redistribute the polarized receptor toward the potential partner (Fig. 2B). Of the $B U D 1^{G 12 V} / B U D 1$ cells that successfully formed zygotes, significantly fewer exhibited gradient tracking behavior as compared to control cells: They either fused with partners positioned near their DS or at the presumptive distal bud site (Fig. 2C) $)^{24,25}$. In the distal-mating class of cells, the receptor polarized directly to the fusion site rather than tracking from the DS toward the mating partner - a phenotype we call jumping ${ }^{21}$.

Given that Bud1 partially rescues DS function in cells co-expressing Bud1 ${ }^{\mathrm{G} 12 \mathrm{~V}}$, as evidenced by the viability of $B U D 1^{G 12 V} / B U D 1$ cells, these data support our hypothesis that Bud1 must be inactivated to allow GTM tracking. 


\section{The receptor polarizes to the DS but fails to track in bem $1^{\triangle C P X}$ cells}

The polarization of RFP-Bud2 to the DS before the receptor in mating cells suggested that Bud1 is inactivated early during GTM assembly. Miller et al demonstrated that inactive Bud1 (hereafter, Bud1 ${ }^{\mathrm{GDP}}$ ) directly interacts with the C-terminal half of the Bem1 Phox homology domain (hereafter, the CPX domain) ${ }^{26}$. In mating cells, the earliest event in GTM assembly detected thus far is the localization of Farl to the DS, presumably in complex with Cdc $24^{20}$. We postulated that Far1-Cdc24 recruitment to the assembly site depends on the reported interaction of Cdc24 with Bem $1^{13-15}$, and further, that Bud1 ${ }^{\text {GDP }}$ initiates GTM assembly by recruiting Bem1 to the DS. To test this conjecture, we took time-lapse images of MATa cells expressing Bem $1^{\triangle \mathrm{CPX}}$ and the receptor reporter in mating mixtures. In the absence of Bud1 ${ }^{\mathrm{GDP}}$-Bem 1 interaction, we expected a failure to recruit Far1-Cdc24, and therefore, no receptor polarization to the DS. Surprisingly, the receptor polarized to the DS in bem $1^{\triangle C P X}$ cells just as well as in the WT cells (Fig. 3). However, bem $1^{\triangle C P X}$ cells showed no evidence of gradient-sensing: their polarized receptor crescents did not track toward potential mating partners, and they invariably shmooed and mated at the DS. These observations suggest that the interaction between inactive Bud1 and Bem1 is not required for GTM assembly, but that the Bem1 CPX domain is required for tracking.

\section{Receptor tracking is defective in exo $70^{4 d C}$ cells}

We have shown that the receptor tracks normally in $b u d 1 \Delta$ cells ${ }^{20}$. Therefore, the inability of the receptor to track in bem $1^{\triangle C P X}$ cells must be because the CPX domain of Bem 1 provides a critical tracking function independent of Bud1, and not because tracking depends on the interaction of Bud1 ${ }^{\mathrm{GDP}}$ with Bem1. We have also shown that tracking correlates with the concentration of the vesicle delivery marker, Sec3, to the upgradient side of the GTM ${ }^{20}$. Notably, 
Bem1 has been reported to direct vesicle delivery independent of actin cables through the interaction of its Phox homology domain with Exo7027.

Sec3 and Exo70 serve as partially redundant "pioneer proteins" for the vesicle-tethering exocyst complex - they position and catalyze assembly of the complex at discrete locations on the $\mathrm{PM}^{28,29}$. Although Sec3 and Exo70 are transported to the PM along with the other components of the complex by Myo2 on actin cables, they are unique in their direct recruitment to the PM independent of F-actin: Sec3 binds to Cdc42 and Rho1 while Exo70 binds to Bem1 ${ }^{28-}$ 31. The C-domain of Exo70 is essential for Exo70-Bem1 interaction and its deletion (denoted $\Delta \mathrm{dC}$ ) diminishes $\mathrm{AI}-\mathrm{VD}$ without affecting $\mathrm{AD}-\mathrm{VD}^{30}$.

To determine whether the failure of the GTM to track in bem $1^{\triangle C P X}$ cells is due to the loss of Bem1-directed AI-VD, we took time-lapse images of MATa exo $70^{\triangle d C}$ cells expressing Ste2-GFP in mating mixtures (Fig. 4A). Consistent with our hypothesis, about $40 \%$ of the exo $70^{4 d C}$ cells ignored MATa cells with which they were in direct contact, a behavior we see ten times less often in WT mating mixtures (Fig. 4B). In most of these cells, the receptor polarized to the DS but did not track toward proximal partners; consequently, such cells shmooed at the DS (Fig. 4A and B). Of the exo $70^{\unlhd d C}$ cells that successfully formed zygotes, a significantly larger fraction mated at their DS or at the presumptive distal bud site as compared to the control cells, while a significantly smaller fraction exhibited gradient tracking and chemotropic mating (Fig. 4C). Given that AI-VD is partially maintained by Sec3 in exo $70^{\Delta d C}$ cells ${ }^{30}$, these data suggest that $\mathrm{AI}-\mathrm{VD}$ is required for tracking. 


\section{Markers for AD-VD do not track with the receptor but polarize strongly at the eventual}

\section{chemotropic site in mating cells}

It has been proposed that the movement of the polarity complex along the cell cortex during yeast gradient sensing is driven by vesicles delivered to the PM on micro actin filaments ${ }^{32,33}$. To determine whether AD-VD is associated with gradient tracking in mating cells, we engineered MATa cells co-expressing the receptor reporter and RFP-tagged Myo2, a marker for vesicles delivered to the PM on actin cables $^{34}$; RFP-tagged Abp1, a marker for actin patches ${ }^{35}$; or RFPtagged Abp140, a marker for actin cables ${ }^{36}$. We took time-lapse images of these MATa cells

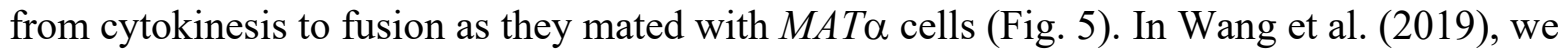
demonstrated that Sec3-RFP robustly polarizes to the DS with the receptor, tracks on the leading side of the receptor crescent (Fig. 6A), and becomes highly concentrated at the eventual CS (Fig. $6 \mathrm{~B})^{20}$. Unlike Sec3-RFP, Myo2-RFP polarized to the DS in only $45 \%$ of the cells examined (23 out of 51 cells) (Fig. 5A) and did not track with the leading peak of receptor (Figs. 5A and 6C). Like Sec3-RFP, Myo2-RFP polarized sharply at the CS (Figs. 5A and 6D). Similarly, we detected actin cables marked by Abp140-RFP oriented toward the receptor at the DS during assembly and at the CS after stabilization, but not during tracking (Fig. 5B). On the contrary, Abp1-RFP polarized to the DS, tracked behind the receptor (Figs. 5C and 6E), and polarized as peaks surrounding the eventual CS (Figs. 5C and 6F). This is consistent with our conclusion, based on time-lapse imaging of Sla1-RFP, that receptor-driven endocytosis is maximal behind the peak of tracking receptor and surrounds the eventual $\mathrm{CS}^{20}$. Together, these observations suggest that AD-VD does not contribute to GTM tracking, but is operative during GTM assembly at the DS and stabilization at the eventual CS. 


\section{The receptor tracks in myo2-16 cells mated with WT $M A T \alpha$ cells at restrictive temperature}

To determine whether AD-VD is required for tracking, we engineered MATa cells expressing Ste2-GFP and the temperature-sensitive allele myo2-16 ${ }^{37}$. Myo2, the type V myosin motor protein in yeast, docks post-Golgi vesicles to actin cables and carries them to sites of actin-dependent polarized secretion on the $\mathrm{PM}^{34}$. At the restrictive temperature of $33^{\circ} \mathrm{C}$, the myo2-16 mutant protein cannot dock post-Golgi vesicles to actin cables but has no effect on actin-cable assembly ${ }^{37}$. As a result, AD-VD is blocked in the mutant cells. As previously reported for other myo2-16 strains $^{37}$, the mutant cells were unable to initiate the growth of a new daughter cell (bud) at the restrictive temperature (Fig. 7A-C). We took time-lapse images of WT MATa cells and myo2-16 MATa cells in mating mixtures at $33{ }^{\circ} \mathrm{C}$ (Fig. 7D-F). Whereas the restrictive temperature had no effect on tracking and mating in WT cells (Fig. 7 D, G, and H), about $40 \%$ of the MATa myo2-16 cells ignored MAT $\alpha$ cells with which they were in direct contact (Fig. 7E and G). Unlike the $B U D 1^{G 12 V} / B U D 1$ and the exo $70^{\triangle d C}$ mutant cells that ignored potential mating partners due to defects in tracking, most of the myo2-16 cells exhibited a defect

in GTM assembly. They were either unable to polarize the receptor to the DS or the polarized receptor signal became undetectable over time. This phenotype is consistent with our observation that Myo2-RFP polarized to the DS in about $45 \%$ of the WT cells, suggesting that AD-VD contributes to GTM assembly. In the cohort of myo2-16 cells that successfully mated, the receptor polarized to the DS, tracked along the PM, and stabilized at the CS shortly before cell fusion, phenocopying receptor behavior in WT cells (Fig. 7F-H). This result demonstrates that blocking AD-VD does not affect tracking. 


\section{DISCUSSION}

Significant progress has recently been made in understanding how mating yeast cells interpret pheromone gradients and accurately position their chemotropic growth sites. Emerging evidence suggests that yeast are not global gradient sensors, able to orient and polarize directly toward their mating partners. Rather, yeast cells become competent to sense pheromone gradients by colocalizing the pheromone-signaling and vesicle-trafficking systems at an intrinsically determined polarity site, the DS - assembling what we call the gradient tracking machine. Within the GTM, the pheromone gradient determines the pattern of dimeric receptor species, which segregates anterograde and retrograde vesicle trafficking, resulting in the redistribution of the GTM toward the pheromone source and stabilization at the $\mathrm{CS}^{20,21}$. At the level of the receptor, $\mathrm{G}$ protein, RGS protein Sst2, and Sec3, GTM redistribution appears to be deterministic: The primary pheromone sensing components and pioneer exocytic marker move together, directly and steadily upgradient from the DS to the CS. This model not only provides a framework for understanding how yeast cells decode shallow pheromone gradients, it also explains how a weak spatial signal can position the CS despite the strong intrinsic polarity of the DS. Instead of a global gradient-sensing mechanism that competes with the DS, a mobile GTM is assembled at the DS, which then incrementally redistributes toward the mating partner. However, concentrating the key polarity and secretory proteins at the DS presents its own problems: What prevents polarized growth at that site? And how is the GTM released for tracking after its assembly is complete? Here we describe two mechanisms that enable tracking (Fig. 8). First, the Ras GTPase Bud1 must be inactivated to allow GTM release. Second, actin-independent - but not actin-dependent - vesicle delivery must be targeted upgradient to drive GTM redistribution. 


\section{The bud-positioning function of the DS must be inactivated to release the GTM}

During the GTM assembly process in cells preparing to mate, we found that the Bud1 GEF became undetectable, whereas the Bud1 GAP polarized to the DS. Subsequently, the Bud1 GAP tracked with the receptor to the CS. These results suggest that Bud1 is inactivated during GTM assembly and that it remains inactive throughout tracking. Is Bud1 inactivation required for tracking to begin? Even when co-expressed with WT Bud1, which is necessary for viability, GTP-locked Bud1 conferred a severe defect in tracking: A large fraction of $B U D 1^{G 12 V / B U D 1}$ cells either continued to bud or shmooed at the DS. Taken together with our previous finding that budl $\Delta$ cells assemble multiple GTMs at random positions on the PM - a maladaptive phenotype that occasionally results in budl $\Delta$ cells fusing with multiple partners and forming heterokaryons rather than zygotes ${ }^{20}$ - we conclude that Bud1 plays an important albeit transitory role in yeast mating. It is initially required to promote assembly of a single GTM at a specific point in the cell cycle (late G1) and at a specific cortical position (the DS). It must then be inactivated and remain inactive to permit tracking. In this view, the Bud1 GAP travels with the GTM to ensure that Bud1 stays off. Without this protection, stochastic activation of Bud1 could trigger positive feedback amplification of the Cdc42-Cdc24-Bem1 loop, leading to local nucleation of actin cables $^{8-12}$. This would likely cause the GTM to stall before aligning with the gradient source, as we observed in $B U D 1^{G 12 V / B U D 1}$ cells.

\section{Actin-independent vesicle delivery is essential for GTM tracking}

Based on the localization of Sec3, a pioneer component of the exocyst complex, we previously concluded that vesicle delivery is involved in all three phases of gradient sensing after global internalization $^{20}$. We showed that Sec3-RFP polarizes to the DS during GTM assembly, concentrates to the upgradient side of the GTM during tracking, and sharply peaks in the center 
of the GTM after stabilization. The observations reported in this paper suggest a relationship between the mode of vesicle delivery and the phase of gradient sensing: Vesicles are delivered to the PM by both actin-cable-dependent and actin-cable-independent mechanisms during GTM assembly and stabilization, but exclusively by an actin-cable-independent mechanism when the GTM is tracking. We infer that the high rate of vesicle delivery along actin cables ${ }^{28}$, which is needed to assemble the GTM and polarize growth at the CS, must be turned off to permit gradient tracking. In other words, there is a tradeoff between faster vesicle delivery and GTM mobility.

In principle, actin-cable-directed secretion could be the primary determinant of GTM behavior or the stability/mobility of the GTM could be the primary determinant of when and where actin cables are nucleated. We favor the latter possibility, "GTM first, cables second." Following pheromone-induced global internalization of the receptor and $\mathrm{G}$ protein, during the 10-15 minute GTM assembly phase, receptor and G protein gradually increase at the $\mathrm{DS}^{20}$. We found that $\mathrm{AD}-\mathrm{VD}$ markers were detectable at the $\mathrm{DS}$ in about half of the cells examined and that blocking AD-VD prevented GTM assembly in about half of the cells examined as well. The simplest way to explain why some cells need F-actin and Myo2 to complete GTM assembly and others do not is variability in how much receptor and G protein remain at the DS after global internalization. Another observation that supports the "GTM first, cables second" view is that we never detected the AD-VD markers at the CS before the GTM began to stabilize.

Our conclusion that AI-VD is both necessary and sufficient to enable tracking is based on the following observations. First, AD-VD is dispensable during tracking and chemotropic mating. Second, the direct interaction between Bem1 and Exo70, which localizes Exo70 (and thus the exocyst complex) to secretion sites on the $\mathrm{PM}^{27}$, is critical for tracking. We 
demonstrated this in two independent experiments by mutating the interaction domain of each partner. Whereas partial deletion of the Bem1 Phox homology domain abolished tracking, deletion of the Exo70 C-domain severely compromised but did not entirely eliminate it. The residual tracking ability of Exo $70^{\Delta \mathrm{dC}}$ cells is attributable to the functional redundancy of Sec3 and Exo70 in driving AI-VD. Unfortunately, because $\sec 3^{\Delta N}$ exo $70^{4 d C}$ double mutant cells are not viable ${ }^{30}$, this explanation cannot be tested.

What directs AI-VD toward the CS in mating cells? According to the model we proposed in Wang et al. ${ }^{20}$, a greater proportion of receptors are activated and protected from endocytosis on the upgradient side of the tracking GTM; consequently, this region is enriched in activated G $\alpha$ and free $G \beta \gamma$. Because free $G \beta \gamma$ interacts directly with the Far1-Cdc24/Bem 1 complex $^{13-15}$, we previously suggested that $\mathrm{G} \beta \gamma$ directs secretion by positioning Far1-Cdc24/Bem1, and thus, Cdc42-Sec $3^{20}$. Although the positioning of Sec3 by G $\beta \gamma$ may play a role in tracking, the results we describe here indicate that the Exo70-Bem1 interaction is essential for this process. Therefore, we propose that G $\beta \gamma$ recruits Exo70 upgradient within the GTM through their mutual interaction with Far1-Cdc24/Bem1 and thereby biases AI-VD toward the gradient source (Fig. 8). It will be interesting to determine whether the pheromone-induced phosphorylation of $\mathrm{G} \beta$ enhances the affinity of $\mathrm{G} \beta \gamma$ for Far1-Cdc24/Bem1, as we have recently shown that phosphorylated G $\beta$ concentrates on the leading side of the GTM and is a directional cue ${ }^{21}$.

Why does the mode of vesicle secretion change as mating cells progress from GTM assembly to tracking and from tracking to stabilization? Robust polarized growth such as bud and shmoo formation requires rapid, focused, and stable vesicle delivery along actin cables. In contrast, gradient tracking requires vesicle delivery at a rate that does not result in polarized growth, as well as dynamic positioning of the secretory site in response to the pheromone 
gradient. AI-VD positioned by a heterotrimeric G protein whose local activity and concentration directly reflect that of the pheromone receptor is likely a faster and more flexible way to effect tracking than a mechanism that depends on the polymerization and depolymerization of actin cables.

What controls the transition from AD-VD at a fixed site during GTM assembly to AI-VDpowered GTM tracking, and back to AD-VD at a fixed site after GTM stabilization? Our results suggest that Bud1 must be inactivated to enable tracking. Because Bud1 positions Far1Cdc24/Bem1-Cdc42 to nucleate actin cables during bud emergence, it likely plays the same role during GTM assembly. As active Bud1 disappears, the Far1-Cdc24/Bem1 complex is freed to interact with $\mathrm{G} \beta \gamma$ and Exo70. In this view, Bud1 inactivation is the switch that turns off AD-VD and allows G $\beta \gamma$-positioned AI-VD to predominate during the transition from assembly to tracking. Our data also indicate that AD-VD markers become detectable again only after the GTM reaches the CS and stabilizes. This observation suggests that actin cables cannot be nucleated if the polarity complexes are mobile. In this view, the positional stability of the GTM determines where and when actin cables will form, thus aligning AD-VD with the pheromone source.

\section{Intrinsic polarity may be integral to the differentiation of many cell types}

Depending on environmental conditions, haploid yeast cells choose one of three distinct fates late in the G1 phase of the cell cycle: When well nourished, they commit to mitosis and begin to polarize the growth of a daughter cell, or bud, at the DS, concomitant with the initiation of $\mathrm{S}$ phase; in mating mixtures, they assemble the GTM at the DS preparatory to locating and fusing with a proximal partner; when starved, they form long, chained projections called pseudohyphae, likely at the DS, which are thought to be used to forage for nutrients ${ }^{38}$. In 
addition to being determined at a unique point in the cell cycle (late G1), we infer that yeast cell fate is regulated at a unique cortical site (the DS). The importance of DS regulation during budding is well documented ${ }^{39}$. The work we have presented here, and previously ${ }^{20}$, demonstrates the essential role of DS regulation during mating. Other studies have shown that yeast cells cannot initiate pseudohyphal growth in the absence of Bud1, Bud2, or Bud5, suggesting that DS regulation is essential for cellular differentiation in response to starvation ${ }^{40}$. Thus, in S. cerevisiae, the specific structure generated at the intrinsically determined polarity site depends on the environmental input.

Like $S$. cerevisiae, most cell types in higher eukaryotes begin to differentiate when their progenitor cells complete a division cycle, and like the DS in yeast, polarity sites on the PM are associated with cell division ${ }^{41}$. Moreover, the basic components and systems required to generate cell polarity and direct vesicle secretion are highly conserved across the eukaryota. Historically, cells were thought to polarize in response to environmental cues as they differentiated ${ }^{41}$. However, recent evidence in many models suggests that the establishment of intrinsic polarity independent of environmental stimuli - precedes and is required for proper differentiation ${ }^{41-45}$. For example, actomyosin flows generate mechanical constraints that result in the establishment of polarity, which subsequently determines cell fate in M. musculus and C. elegans ${ }^{42,43}$; during inner-outer lineage differentiation at the eight-cell stage of mice embryonic development, F-actin and polarity-related proteins such as PKC, PARs, and Ezrin are gradually translocated from the division plane to the apical cortex of the outer cells before fate determination ${ }^{44}$; and, human pluripotent stem cells autonomously develop polarity before differentiating ${ }^{45}$. It remains to be seen whether these and other differentiating cell types use division-marked polarity site(s) to assemble protein complexes that enable them to respond to environmental cues. If so, it will be 
interesting to determine whether these protein complexes relocate to environmentally determined positions, how the pre-existing polarity sites are regulated, and whether different modes of vesicle delivery are involved in these processes.

\section{MATERIALS AND METHODS}

Molecular and microbiological techniques. Standard methods were used for microbial culture and molecular manipulation, performed as described previously ${ }^{46-48}$.

Yeast strain construction. The yeast strains used in this study are listed in Table S1. They were derived by transformation of strain 15Dau (MATa ade1 his2 leu2-3,-112 trp1 ura3A), which is congenic with strain BF264-15D ${ }^{49}$. In situ-tagged strains XWY143, XWY145, XWY164, XWY169, XWY176, XWY180, XWY184, and XWY188 were generated by integrating Bsu36I-cut XWB121, XbaI-cut XWB123, PstI-cut XWB143, BlpI-cut XWB148, SalI-cut XWB156, SalI-cut XWB162, StuI-cut XWB163, or BlpI-cut XWB182 into strain XWY065, respectively. Strain XWY185 was generated by transforming XWB173 into XWY065. All genomic modifications were confirmed by sequencing (UIC Research Resource Center Sequencing Core).

Plasmid construction. The plasmids used in this study are listed in Table S2. All genes were directly amplified from the DSY129 genomic DNA except for the gene encoding the RFP protein, which was amplified from DSB405. XWB121, YIplac211-Pbud2-RFP-BUD2 ${ }^{1-405}$ was constructed by PCR-amplifying the BUD2 promoter with the oligos 5'-ATCAGAATTCTATCAATGCTAGCGTTGAGATGT-3' and 5'-ATCAGGTACCAATATGATACAATCAAAGATAAACACCACG-3', the RFP fragment with the oligos 5'-ATCGGGTACCATGGTTTCAAAAGGTGAAGAAGATAATATG-3' and 5'-ATCAGTCGACTTTATATAATTCATCCATACCACCA- 
GTTG-3', and BUD2 bases 1-405 with the oligos 5'-ATCAGTCGACATGAGCTCCAACAATGAACC-3' and 5'-CAGTAAGCTTGATCAGACATGTTAGCA-ATTTCTTG-3'. The EcoRIand KpnI-digested Pbud2 PCR product, the KpnI- and SalI-digested RFP PCR product, and the SalI- and HindIII-digested BUD2 ${ }^{1-405}$ PCR product were inserted into YIplac211. XWB123, YIplac211-BUD5 ${ }^{1141-1926-R F P}$ was constructed by PCR-amplifying BUD5 bases 1141-1926 with the oligos 5'-ATCAGCATGCGATGAATTGTGCGGTGCAAC-3' and 5'-ATCAGGTACCGGTAAGCCTTGGAACCTTAGC-3', and RFP fragment with oligos 5'-ATCGGGTACCATGGTTTCAAAAGGTGAAGAAGATAATATG-3' and 5'-ATCAGAATTCTTATTTATATAATTCATCCATACCACCAGTTG-3'. The SphI- and KpnI-cut $B U D 5^{1141-1926}$ PCR product and the KpnIand EcoRI-digested RFP PCR product were inserted into YIplac211. XWB143, YIplac211-BEM1 ${ }^{649-1032 ; ~ 1225-1656, ~ w a s ~ c o n s t r u c t e d ~ b y ~ P C R-a m p l i f y i n g ~ B E M I ~ b a s e s ~ 649-1032 ~}$ with oligos 5'-CAGTAAGCTTATTGCCACGGGGTACGCAACAGGTAAC-3' and 5'-TCCACCTGCATCCCTCAACTTACCGGCC-3' and BEMI bases 1225-1656 with oligos 5'-GAAAGAGACGAAAATCAAAACAATATTAAAAC-3' and 5'-ATCAGAATTCTCAAATATCGTGAACGGAAATTTTCAG-3'. The HindIII-digested $B E M 1^{649-1032}$ PCR product and the EcoRIdigested $B E M 1^{1225-1656} \mathrm{PCR}$ product were inserted into YIplca211. XWB148, YIplac211MYO2 ${ }^{4261-4722}$-RFP, was constructed by PCR-amplifying MYO2 bases 4261-4772 with oligos 5'-CAGTAAGCTTGAGGAATGGTGCAAGACGCATGG-3' and 5'-ATCGGGATCCGTGGCCGTCTTGAACGACTTG-3', and RFP fragment with oligos 5'-ATCGGGATCCATGGTTTCAAAAGGTGAAGAAGATAATATG-3' and 5'-ATCAGAATTCTTATTTATATAATTCATCCATACCACCAGTTG-3'. The HindIII- and BamHI-digested $\mathrm{MYO}^{4261-4772} \mathrm{PCR}$ product and the BamHI- and EcoRI-digested RFP PCR product were inserted into YIplac211. XWB156, YIPlac211-ABP1 ${ }^{1414-1776}$-RFP, was constructed by PCR-amplifying ABP1 bases 1411-1776 with 
oligos 5'-ATCGCCCCAAGCTTACCTTCTAG-3' and 5'-ATCAGGTACCGTTGCCCAAAGACACATAATTGC-3', and RFP fragment as described in XWB123. The HindIII- and KpnIdigested $A b p 1^{1141-1776}$ PCR product and the KpnI- and EcoRI-digested RFP PCR product were inserted into YIplac211. XWB162, YIplac211-ABP140 $0^{610-1885}$-RFP, was constructed by PCRamplifying $A B P 140$ bases 610-1885 with oligos 5'-CAGTAAGCTTGCTAATGATGGCTCTACAAGTACC-3' and 5'-ATCAGGTACCTTGAGGAACGTCAAACACAGC-3', and RFP fragment as described in XWB123. The HindIII- and KpnI-digested $A b p 140^{610-1885} \mathrm{PCR}$ product and the KpnI- and EcoRI-digested RFP PCR product were inserted into YIplac211. XWB163, YIPlac204-myo2 ${ }^{3388-4475}$, by PCR-amplifying MYO2 bases 3388-4475 with oligos 5'-CAGTGTCGACTGATGCTTGAGAATTCCGACTTATCTCC-3' and 5'-ATCGGGATCCTTAGTGGCCGTCTTGAACGACTTG-3'. The underlined sequence indicates the additional stop codon introduced after base 4475 for generating the myo2-16 mutant. The SalI- and BamHI-digested was inserted into YIplac204. XWB171, YCplac33-Pbud1-BUD1 was generated by PCR amplifying the $B U D 1$ promoter and $B U D 1$ with oligos 5'-TTTCTCGAATTCTCAGGTAGTACTG-3' and 5'-CAGTAAGCTTCTATAGAATAGTGCAAGTGGAAGCG-3'. The EcoRI- and HindIII-digested PCR product was inserted into YCplac33. XWB173, YCplac33-Pbud1$\mathrm{BUD}^{\mathrm{G} 12 \mathrm{~V}}$, was generated by sited directed mutagenize XWB171 using oligos 5'-GTAGTATTGGGTGCTGTTGGTGTCGGTAAATCCTGC-3' and 5'-GCAGGATTTACCGACACCAACAGCACCCAATACTAC-3'. The underlined bases indicate the point mutation introduced by this mutagenesis. XWB182, YIPlac211-Exo70 ${ }^{715-1035-l i n k e r-1546-1872}$, was generated by PCR amplifying the EXO70 bases 715-1035 using oligos 5'-ATCAGGTACCATGAACAGCTACACGGAGGCC-3' and 5'- GAATAATGACTGTGTTACTTGACGTACTTCTTG-3', and EXO70 bases 1546-1872 using oligos 5'-GTGGGTACCGGTTCGGGTGTATCAGATTGGAG- 
AGATTTAACTGC-3' and 5'- ATCAGAATTCCTATCTCACTAATTGGTTAAGAACAGT-

AG-3'. The underlined sequence indicates the peptidyl linker introduced for proper Exo70 function. The KpnI-digested $E X O 70^{715-1035} \mathrm{PCR}$ product and the EcoRI-digested $E X O 70^{1546-1872}$ PCR product were inserted into YIplca211.

Time-lapse imaging of mating mixtures. The time-lapse imaging experiments were performed as described in Wang et al. ${ }^{20}$. WT MATa cells and experimental MATa cells were grown to mid$\log$ phase in synthetic $2 \%$ dextrose medium at $30^{\circ} \mathrm{C}$, mixed $1: 1$, and spread at a density of 14,000 cells $/ \mathrm{mm}^{2}$ on agarose pads made from synthetic dextrose medium. Mating mixtures were maintained at $30^{\circ} \mathrm{C}$ using a DeltaVision environment control chamber except as noted below. DIC and fluorescent images were acquired from 12 fields at 2- or 5-minute intervals using a DeltaVision Elite Deconvolution Microscope (GE Healthcare Bio-Sciences) with a 60x oil immersion objective and a Front Illuminated sCMOS camera. To minimize phototoxicity, $5 \mathrm{z}$-sections $0.5 \mu \mathrm{m}$ apart were acquired around the center slice of each cell at each time point. Identical light-emitting diode intensities and exposure times were used to image cells expressing the GFP-tagged reporters (10\% maximum intensity at $461-489 \mathrm{~nm}$ for $200 \mathrm{msec})$ and the RFPtagged reporters (10\% maximum intensity at $529-556 \mathrm{~nm}$ for $200 \mathrm{msec})$. Representative fluorescent images were deconvolved using Huygens Essential software (Scientific Volume Imaging) in standard mode. To image the temperature sensitive myo2-16 strain, the cells were grown to mid-log phase at $25^{\circ} \mathrm{C}$ and incubated at $33^{\circ} \mathrm{C}$ for 30 mins before mixing and spreading onto the agarose pads. Mating mixtures were maintained at $33^{\circ} \mathrm{C}$.

Image analysis. For the two-reporter plots (Fig. 6), PM fluorescence signal intensities were quantified by tracing the circumference of the center-slice DIC images using the segmented line tool of ImageJ; pixel values were determined from the corresponding raw fluorescent images 
after subtracting the background. Cell circumferences were normalized to 100 points and the mean distribution of the receptor was generated by aligning the leading Ste2-GFP peaks with each other during tracking and with the center of the fusion site at the prezygote stage. To determine the proportion of cells that ignored a potential partner and failed to mate (Figs. 2C and 7B), all MATa cells directly in contact with one or more MAT $\alpha$ cell(s) were scored for mating.

Supplemental Material. Table S1 lists the yeast strains used in this study. Table S2 lists the plasmids used in this study.

Table S1. Yeast strains used in this study.

\begin{tabular}{|c|c|c|c|}
\hline Strain & Background & Genotype & Source \\
\hline DSY129 & BF264-15D & MATa ade1 his2 leu2-3,112 trp1-1a ura34 & Stone lab \\
\hline RDY186 & BF264-15D & MATa adel his2 leu2-3,112 trp1-1a ura34 & Stone lab \\
\hline XWY065 & BF264-15D & $\begin{array}{l}\text { MATa adel his2 leu2-3,112 trp1-1a ura34 } \\
\text { STE2-GFP-LEU2 }\end{array}$ & Stone $1 \mathrm{ab}{ }^{20}$ \\
\hline XWY143 & BF264-15D & $\begin{array}{l}\text { MATa adel his2 leu2-3,112 trp1-1 a ura34 } \\
\text { STE2-GFP-LEU2, RFP-Bud2-URA3 }\end{array}$ & This study \\
\hline XWY145 & BF264-15D & $\begin{array}{l}\text { MATa adel his2 leu2-3,112 trp1-1a ura34 } \\
\text { STE2-GFP-LEU2, BUD5-RFP-URA3 }\end{array}$ & This study \\
\hline XWY164 & BF264-15D & $\begin{array}{l}\text { MATa adel his2 leu2-3,112 trpl-1a ura34 } \\
\text { STE2-GFP-LEU2, bem }{ }^{\triangle C P X}-U R A 3\end{array}$ & This study \\
\hline XWY169 & BF264-15D & $\begin{array}{l}\text { MATa adel his2 leu2-3,112 trp1-1a ura34 } \\
\text { STE2-GFP-LEU2, MYO2-RFP-URA3 }\end{array}$ & This study \\
\hline XWY176 & BF264-15D & $\begin{array}{l}\text { MATa adel his2 leu2-3,112 trp1-1a ura34 } \\
\text { STE2-GFP-LEU2, ABP1-RFP-URA3 }\end{array}$ & This study \\
\hline XWY180 & BF264-15D & $\begin{array}{l}\text { MATa adel his2 leu2-3,112 trp1-1a ura34 } \\
\text { STE2-GFP-LEU2, ABP140-RFP-URA3 }\end{array}$ & This study \\
\hline XWY184 & BF264-15D & $\begin{array}{l}\text { MATa adel his2 leu2-3,112 trp1-1a ura34 } \\
\text { STE2-GFP-LEU2, myo2-16-TRP1 }\end{array}$ & This study \\
\hline XWY185 & BF264-15D & 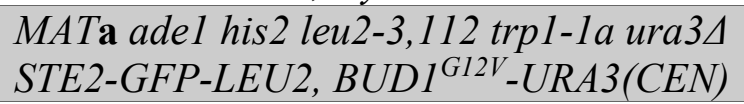 & This study \\
\hline XWY188 & BF264-15D & $\begin{array}{l}\text { MATa adel his2 leu2-3,112 trp1-1a ura3A } \\
\text { STE2-GFP-LEU2, EXO70 } \triangle C P X-U R A 3\end{array}$ & This study \\
\hline
\end{tabular}


Table S2. Plasmids used in this study.

\begin{tabular}{|c|c|c|c|}
\hline Plasmid \# & Plasmid construction & Marker/type & Source \\
\hline DSB405 & pRS406/RFP-BUD1 & URA3/INT & Stone lab \\
\hline XWB121 & YIplac211-Pbud2-RFP-BUD2 ${ }^{1-405}$ & URA3/INT & This study \\
\hline XWB123 & YIplac211-BUD5 ${ }^{1141-1926}-$ RFP & URA3/INT & This study \\
\hline XWB143 & YIplac211-BEM1 649-1032; 1225-1656 & URA3/INT & This study \\
\hline XWB148 & YIplac211-MYO2 ${ }^{4261-4722-R F P}$ & URA3/INT & This study \\
\hline XWB156 & YIPlac211-ABP1 ${ }^{1414-1776}$-RFP & URA3/INT & This study \\
\hline XWB162 & YIplac211-ABP140 $1010-1885$-RFP & URA3/INT & This study \\
\hline XWB163 & YIPlac204-myo $2^{3388-4475}$ & TRP1/INT & This study \\
\hline XWB171 & YCplac33-Pbud1-BUD1 & URA3/CEN & This study \\
\hline XWB173 & YCplac33-Pbud1-BUD1 ${ }^{\mathrm{G} 12 \mathrm{~V}}$ & URA3/CEN & This study \\
\hline XWB182 & YIPlac211-Exo70715-1035-linker-1546-1872 & URA3/INT & This study \\
\hline
\end{tabular}

\section{ACKNOWLEDGEMENTS}

We thank Rob Arkowitz, Edward Draper, Holly Stratton, and members of the Stone lab for helpful discussions and critical reading of the manuscript. Supported by NSF grant 1818067 (DES). The authors declare no competing financial interests. 

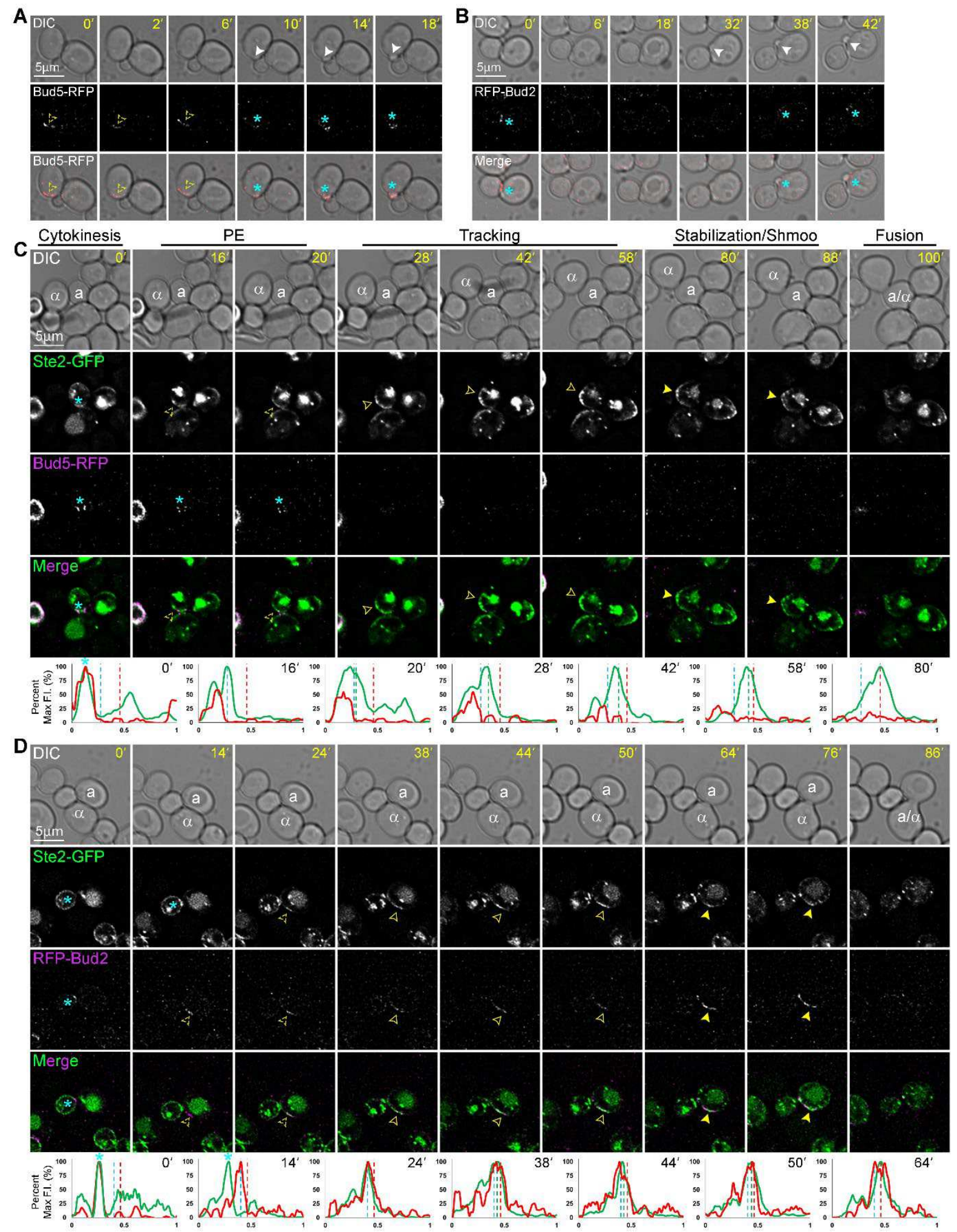


\section{Figure 1. Localization of Bud5-RFP and RFP-Bud2 in vegetative and mating cells.}

(A-D) Representative time-lapse images. MATa cells co-expressing in situ-tagged Ste2-GFP and Bud5-RFP or RFP-Bud2 were imaged during vegetative growth or mixed with an equal number of MAT $\alpha$ cells and imaged from cytokinesis to fusion. The mating partners are labeled $\mathbf{a}$ and $\alpha$ in the DIC images. The blue asterisk indicates reporter localization at the bud neck; dashed arrowheads indicate polarity establishment (PE) at the DS and mark the signal peak; closed arrowheads indicate redistribution and mark the leading peak; filled arrowheads indicate stabilization at the CS and mark the signal peak of Ste2-GFP (the receptor). The plots show the distribution of each reporter on the PM at the indicated time points (10-point rolling average). The x-axes represent distance along the PM; the y-axes indicate the percent fluorescence intensity (F.I.) normalized to the maximum peak intensity for each reporter. The dashed blue, green and red lines mark the DS peak, the leading peak, and the CS peak, respectively. Localization of (A) Bud5-RFP in a vegetative cell; (B) RFP-Bud2 in a vegetative cell; (C) Ste2-GFP and Bud5-RFP in a mating cell; (D) Ste2-GFP and RFP-Bud2 in a mating cell. 

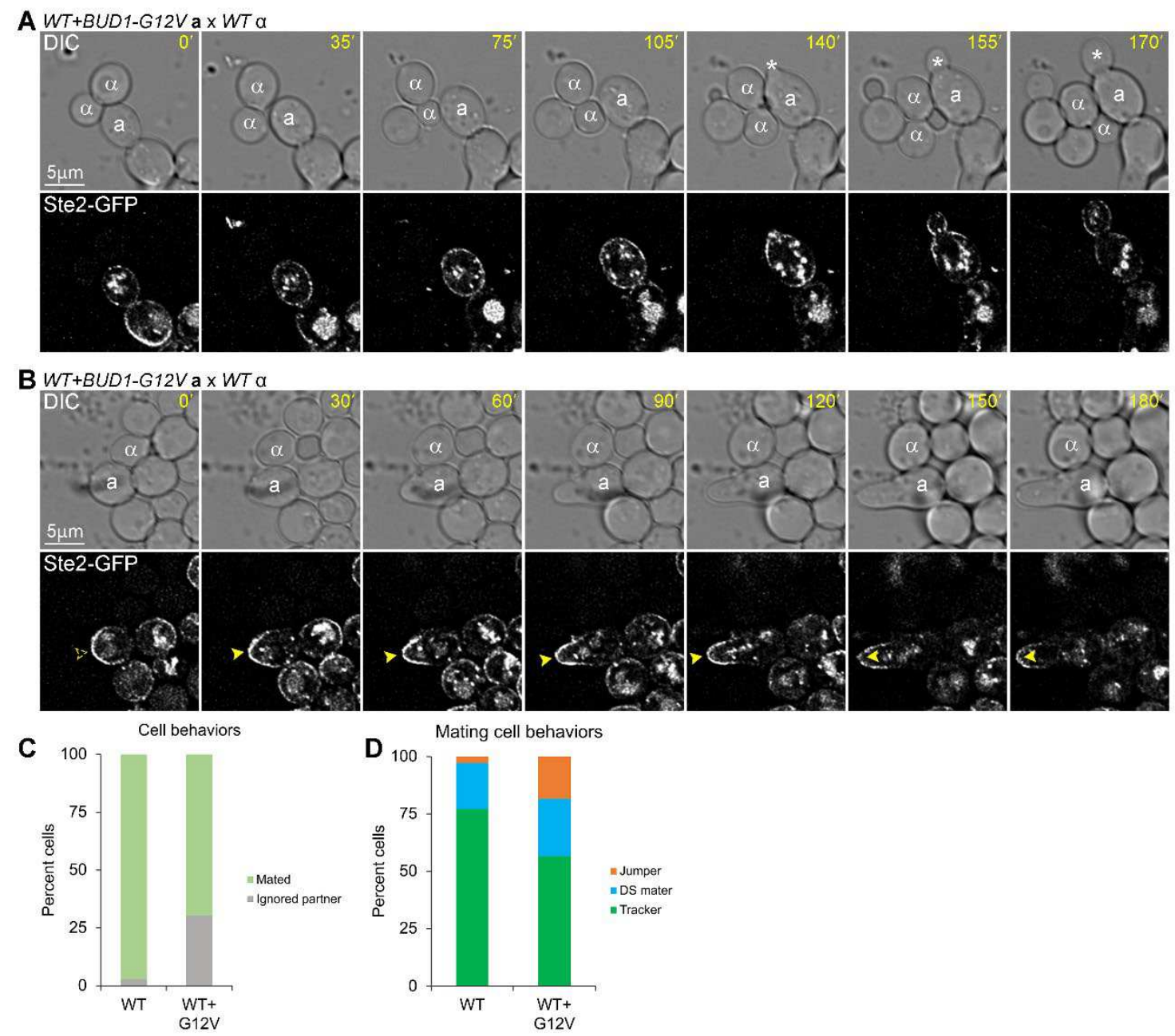

Figure 2. Localization of the receptor in mating cells expressing both Bud1 and Bud1 ${ }^{\mathrm{G12}}$.

(A, B) MATa BUD1 cells co-expressing in situ-tagged Ste2-GFP and Bud1 ${ }^{\mathrm{G} 12 \mathrm{~V}}$ from a centromeric plasmid were mixed with an equal number of $M A T \alpha$ cells and imaged at five-minute intervals. The potential mating partners are labeled as a and $\alpha$ in the DIC images. The dashed arrowheads indicate polarity establishment; filled arrowheads indicate shmoo formation.

Representative images of (A) a MATa cell that ignored the potential mating partner and kept budding; (B) a MATa cell that polarized its receptor but failed to track to the partner. (C) Bar graph showing the proportion of WT cells and cells expressing Bud1 ${ }^{\mathrm{G} 12 \mathrm{~V}}$ that ignored a potential mating partner. $\mathrm{n}=171$ for $\mathrm{WT}$ and 109 for $\mathrm{WT}+\mathrm{Bud}^{\mathrm{G} 12 \mathrm{~V}}$ from two independent experiments; 
$\mathrm{p}<0.0001$. (D) MATa BUD1 cells expressing Bud1 ${ }^{\mathrm{G} 12 \mathrm{~V}}$ are less likely than WT control cells to exhibit gradient-tracking behavior. MATa cells from (B) that completed cytokinesis and formed zygotes were scored as having mated at the default polarity site (DS maters), tracked to a chemotropic site (trackers), or localized directly to the fusion site (jumpers) based on the spatiotemporal dynamics of the Ste2-GFP reporter in time-lapse fluorescent images. $n=100$ for WT cells and 76 for WT + Bud $1^{\mathrm{G} 12 \mathrm{~V}}$ cells. $\mathrm{p}<0.0001$ for percent tracking and percent jumping; $\mathrm{p}=0.21$ (not significant) for percent default mating. 


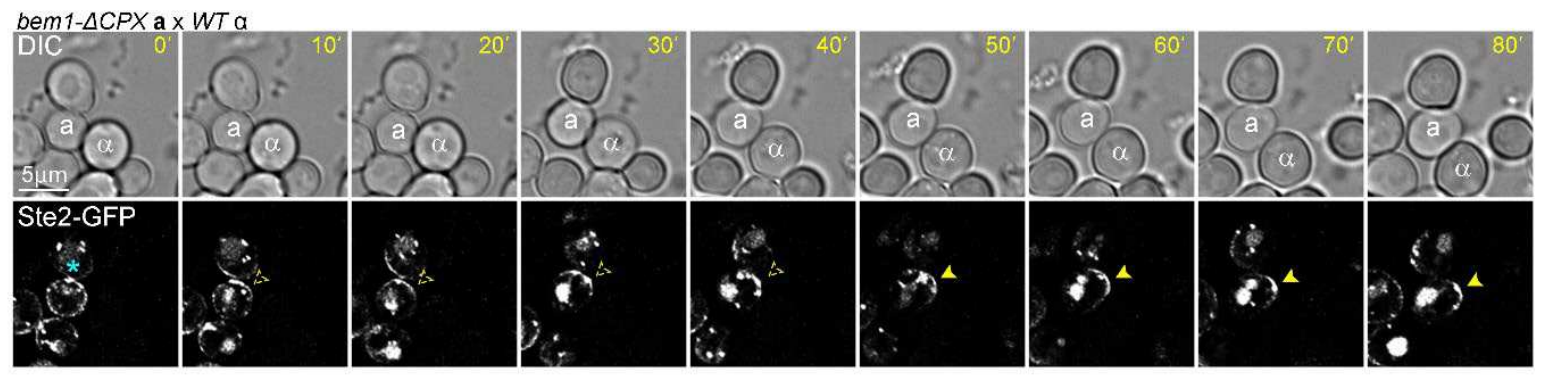

Figure 3. Localization of the receptor in mating Bem $1^{\triangle \mathrm{CPX}}$ cells. MATa bem $1^{\triangle C P X}$ cells expressing in situ-tagged Ste2-GFP were mixed with an equal number of $M A T \alpha$ cells and imaged at five-minute intervals. Representative DIC and fluorescent images are shown. The potential mating partners are labeled as a and $\alpha$ in the DIC images. The blue asterisk indicates receptor localization at the bud neck; dashed arrowheads indicate polarity establishment of the receptor at the DS; filled arrowheads indicate stabilization of the receptor and shmoo formation. 


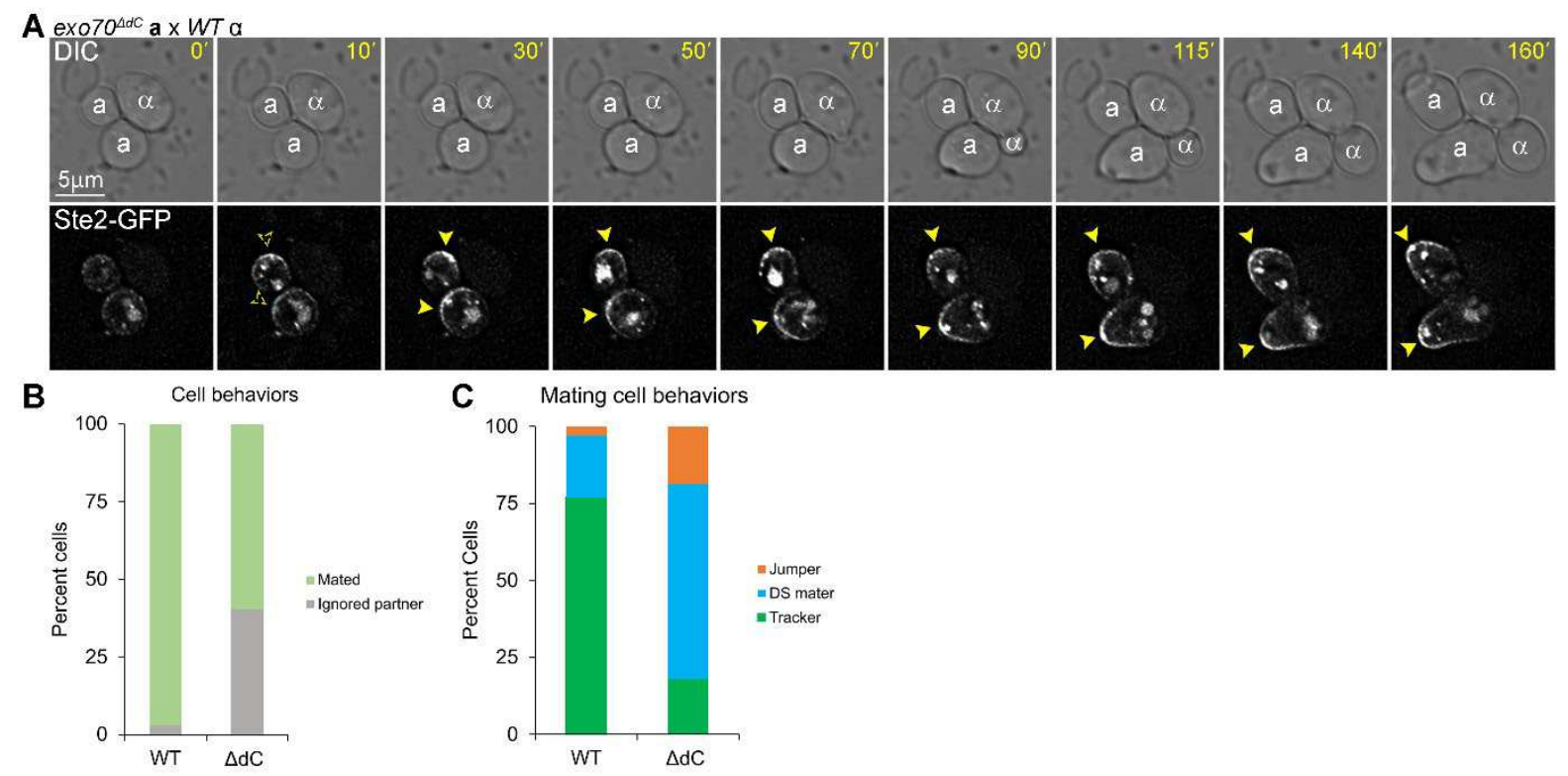

Figure 4. Localization of Ste2-GFP in exo $70^{\Delta d C}$ cells in mating mixtures. (A) MATa exo $70^{\triangle d C}$ cells expressing in situ-tagged Ste2-GFP were mixed with an equal number of MATa cells and imaged at five-minute intervals. The potential mating partners are labeled as a and $\alpha$ in the DIC images. The dashed arrowheads indicate polarity establishment; filled arrowheads indicate stabilization of the receptor and shmoo formation. Representative images show two MATa exo $70^{\triangle d C}$ cells in which the receptor polarized but failed to track to the adjacent MAT $\alpha$ cell. (B) Bar graph showing the proportion of WT and exo $70^{\lrcorner d C}$ cells that ignored a potential mating partner. $\mathrm{n}=171$ for WT and 242 for exo $70^{\triangle d C}$ from two independent experiments; $\mathrm{p}<0.0001$. (C) MATa exo $70^{\unlhd d C}$ cells are less likely than WT control cells to exhibit gradient-tracking behavior. MATa cells from (B) that completed cytokinesis and formed zygotes were scored as having mated at the default polarity site (DS maters), tracked to a chemotropic site (trackers), or localized directly to the fusion site (jumpers) based on the spatiotemporal dynamics of the Ste2GFP reporter in time-lapse fluorescent images. $\mathrm{n}=100$ for WT and 123 for exo $70^{\lrcorner d C} ; \mathrm{p}<0.0001$ for percent tracking, percent jumping, and percent default mating. 

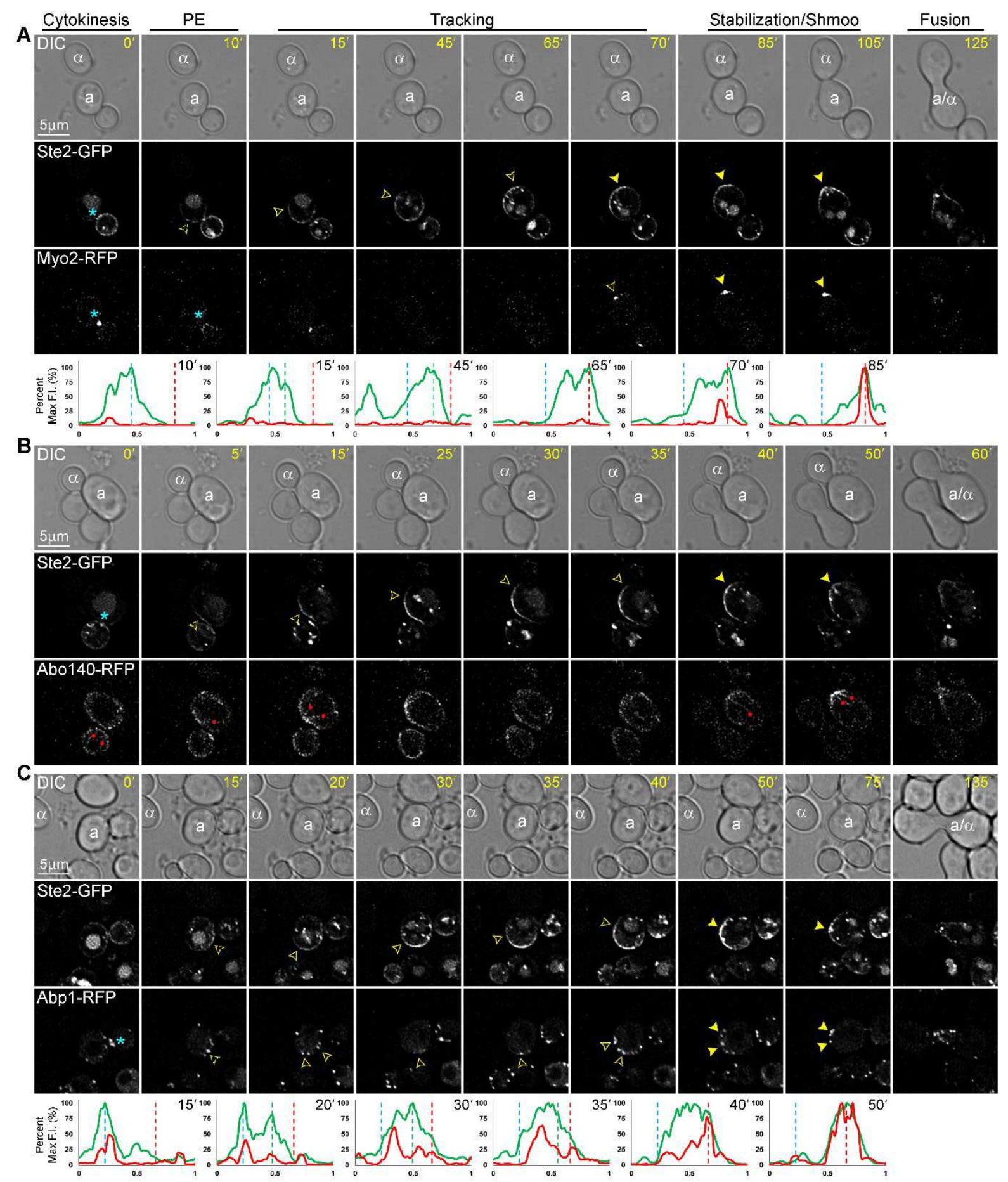

Figure 5. Localization of Myo2-RFP, Abp140-RFP, and Abp1-RFP in mating cells. (A-

C) Representative time-lapse images. MATa cells co-expressing in situ-tagged Ste2-GFP and Myo2-RFP, Abp140-RFP, or Abp1-RFP were mixed with an equal number of MATa cells and imaged from cytokinesis to fusion. The mating partners are labeled a and $\alpha$ in the DIC images. 
The blue asterisk indicates reporter localization at the bud neck; dashed arrowheads indicate polarity establishment (PE) at the DS and mark the signal peak; closed arrowheads indicate redistribution and mark the leading peak; filled arrowheads indicate stabilization at the CS and mark the signal peak of Ste2-GFP; the red dots mark the actin cables. The plots show the distribution of each reporter on the PM at the indicated time points (10-point rolling average). The $\mathrm{x}$-axes represent distances along the PM; the y-axes indicate the percent fluorescence intensity (F.I.) normalized as described in the Materials and Methods. The dashed blue, green, and red lines mark the DS peak, the leading peak, and the CS peak, respectively. Localization of (A) Ste2-GFP and Myo2-RFP in a mating cell; (B) Ste2-GFP and Abp140-RFP in a mating cell; (C) Ste2-GFP and Abp1-RFP in a mating cell. 

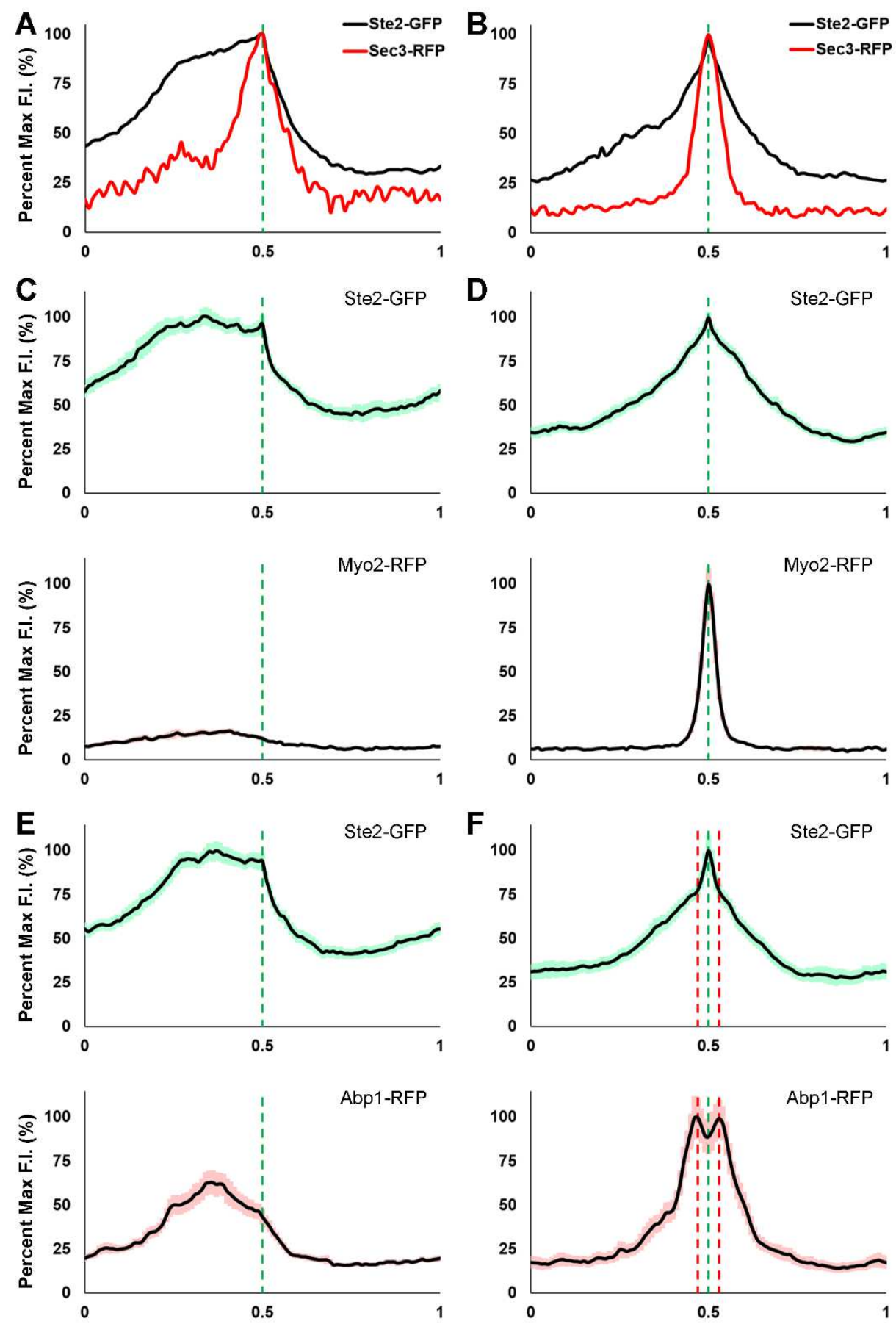

Figure 6. Average PM distribution of Myo2-RFP and Abp1-RFP relative to Ste2-GFP in mating cells during tracking and in shmoos. (A, B) Illustration of Sec3-RFP distribution relative to the receptor distribution during tracking $(\mathrm{A})$ and in shmoos $(\mathrm{B})^{20}$. (C-F) The PM signals of 30 cells two time points before stabilization (tracking) and two time points before 
fusion (shmooing) were quantified with ImageJ, normalized for cell size, and averaged as described in Material and Methods. The plots show the mean signal distribution $\pm \operatorname{SEM}$ (light shadow) of Ste2-GFP (green) and Myo2-RFP (red) or Abp1-RFP (red). The dashed green lines mark the leading receptor peak and the shmoo tip during tracking and in shmoos; the dashed red lines mark the surrounding peaks of Abp1-RFP in shmoos. Average distributions of (C) Ste2GFP and Myo2-RFP during tracking; (D) Ste2-GFP and Myo2-RFP in shmoos; (E) Ste2-GFP and Abp1-RFP during tracking; (F) Ste2-GFP and Abp1-RFP in shmoos. 


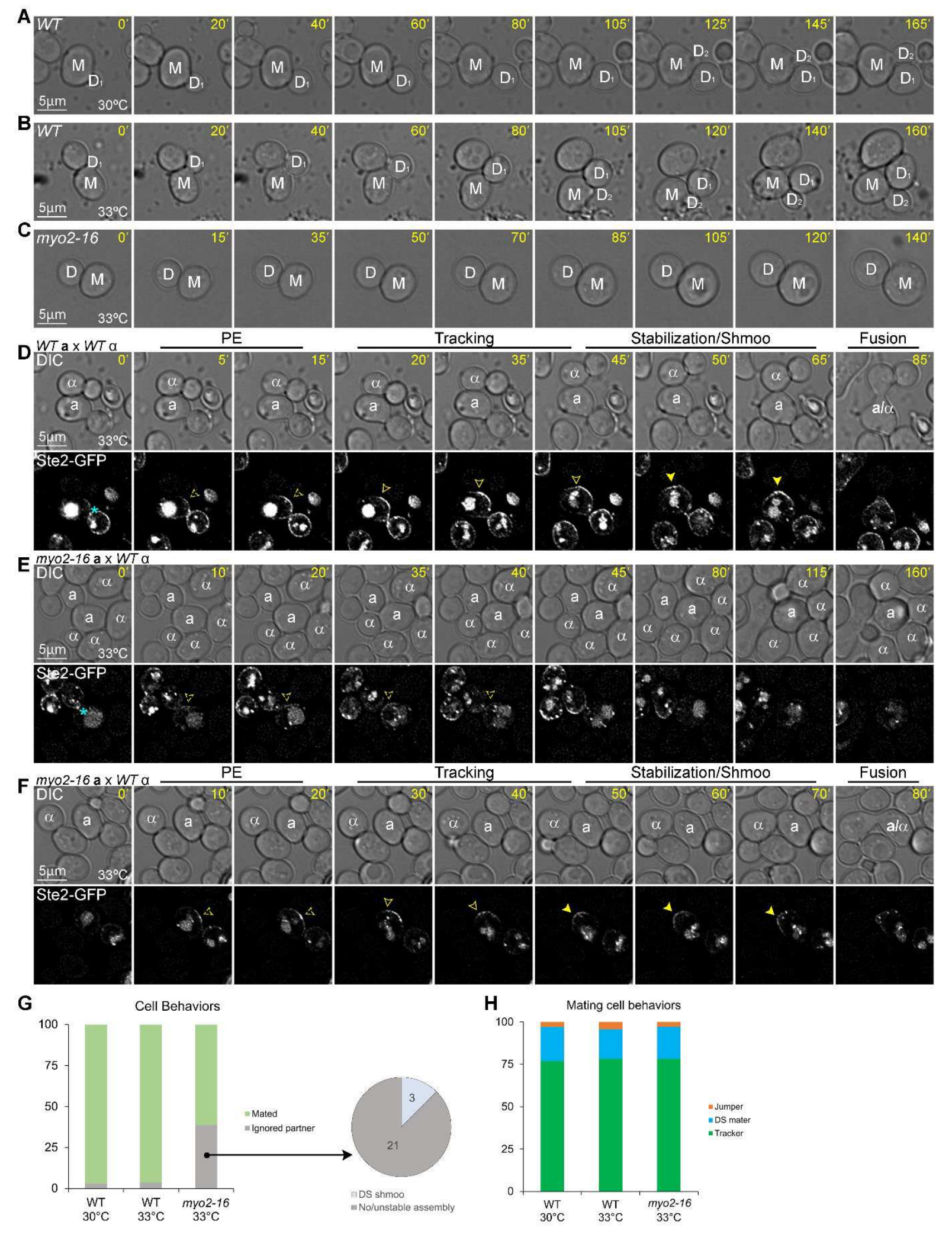




\section{Figure 7. Localization of Ste2-GFP in myo2-16 mutant cells mated at restrictive}

temperature. (A-C) Representative time-lapse images of vegetative cells at the myo2-16

permissive and restrictive temperatures. Mother and daughter cells are labeled M and D. DIC images of (A) a WT cell at $30^{\circ} \mathrm{C},(\mathrm{B})$ a WT cell at $33^{\circ} \mathrm{C}$, and $(\mathrm{C})$ a $m y o 2-16$ cell at $33^{\circ} \mathrm{C}$.

(D-F) Representative time-lapse images of cells in mating mixtures at $33^{\circ} \mathrm{C} \cdot \mathrm{MATa} M Y O 2(\mathrm{WT})$ and MATa myo2-16 mutant cells expressing Ste2-GFP were mated at the restrictive temperature of $33^{\circ} \mathrm{C}$. Potential mating partners are labeled $\mathbf{a}$ and $\alpha$ in the DIC images. The blue asterisk indicates receptor localization at the bud neck; dashed arrowheads indicate polarity establishment (PE) at the DS and mark the signal peak; closed arrowheads indicate redistribution and mark the leading peak; filled arrowheads indicate stabilization at the CS and mark the signal peak of Ste2-GFP. Representative images of (D) a mating WT cell, (E) a myo2-16 cell ignoring potential partners in contact with it, and (F) a mating myo2-16 cell. (G) Bar graph showing the proportion of WT and myo2-16 cells that mated with an adjacent $M A T \alpha$ cell. $\mathrm{n}=171$ for WT at $30^{\circ} \mathrm{C}$, 56 for $\mathrm{WT}$ at $33^{\circ} \mathrm{C}$, and 62 for myo $2-16$ at $33^{\circ} \mathrm{C}$ from two independent experiments; no significant difference when comparing WT at $30^{\circ} \mathrm{C}$ with WT at $33^{\circ} \mathrm{C} ; \mathrm{p}<0.0001$ when comparing myo2-16 to WT at $30^{\circ} \mathrm{C}$ or $33^{\circ} \mathrm{C}$. The pie graph shows the behaviors of the myo2-16 cells that ignored potential mating partners. (H) MATa myo2-16 cells and WT control cells exhibit indistinguishable gradient-tracking behavior. MATa cells from (B) that completed cytokinesis and formed zygotes were scored as having mated at the default polarity site (DS maters), tracked to a chemotropic site (trackers), or localized directly to the fusion site (jumpers) based on the spatiotemporal dynamics of the Ste2-GFP reporter in time-lapse fluorescent images. $\mathrm{n}=100$ for $\mathrm{WT}$ at $30^{\circ} \mathrm{C}, 46$ for $\mathrm{WT}$ at $33^{\circ} \mathrm{C}$, and 37 for myo2-16 at $33^{\circ} \mathrm{C}$; no significant 
difference when comparing any two of these cells for percent tracking, percent jumping, and percent default mating. 


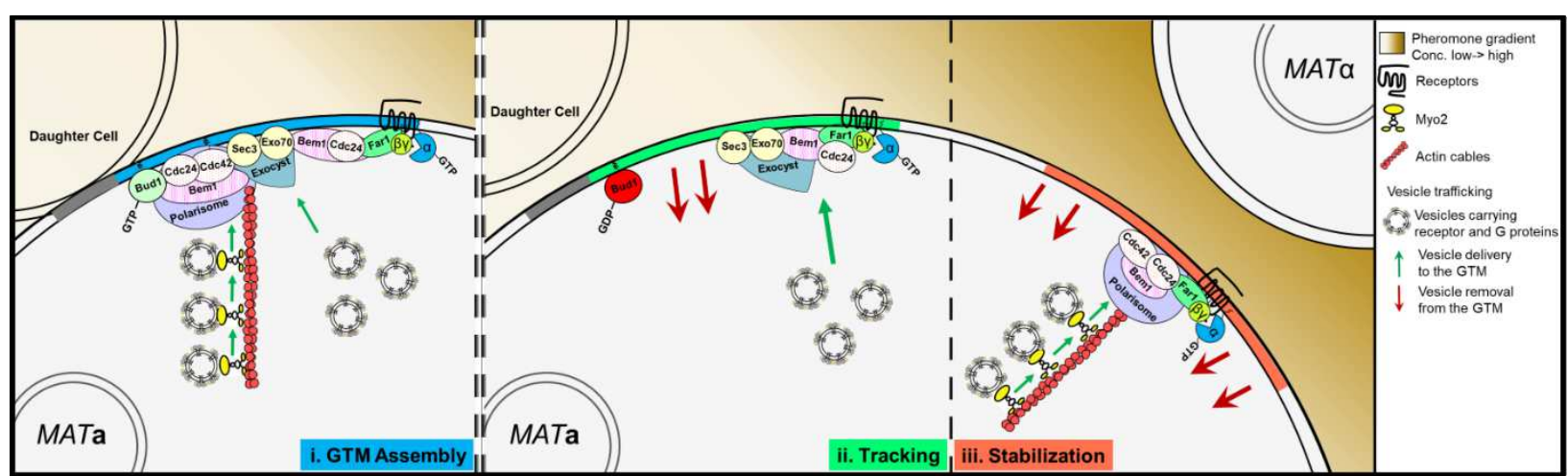

Figure 8. The mode of vesicle delivery controls GTM mobility. (i) During assembly, vesicles carrying the receptor and $\mathrm{G}$ proteins are delivered to the active Bud1-marked DS along and independent of actin cables. (ii) Inactivation of Bud1 and cessation of AD-VD is essential for tracking to start. During tracking, AI-VD is biased upgradient within the GTM, where the active receptor and $\mathrm{G}$ protein are enriched. The interactions between free G $\beta \gamma$ and Far1-Cdc24/Bem1, and Bem1 and Exo70, direct exocyst activity and vesicle fusion up the gradient independent of actin cables. (iii) When the GTM stabilizes at the CS, actin cables are nucleated and robust vesicle delivery drives polarized growth and cell fusion. 


\section{REFERENCES}

1. Iijima, M., Huang, Y.E. \& Devreotes, P. Temporal and spatial regulation of chemotaxis. Dev Cell 3, 469-478 (2002).

2. Hong, K. \& Nishiyama, M. From guidance signals to movement: signaling molecules governing growth cone turning. Neuroscientist 16, 65-78 (2010).

3. Tojima, T., Hines, J.H., Henley, J.R. \& Kamiguchi, H. Second messengers and membrane trafficking direct and organize growth cone steering. Nat Rev Neurosci 12, 191-203 (2011).

4. Arkowitz, R.A. Chemical gradients and chemotropism in yeast. Cold Spring Harb Perspect Biol 1, a001958 (2009).

5. Chant, J. \& Pringle, J.R. Patterns of bud-site selection in the yeast Saccharomyces cerevisiae. J Cell Biol 129, 751-765 (1995).

6. Roemer, T., Madden, K., Chang, J. \& Snyder, M. Selection of axial growth sites in yeast requires Ax12p, a novel plasma membrane glycoprotein. Genes Dev 10, 777-793 (1996).

7. Marston, A.L., Chen, T., Yang, M.C., Belhumeur, P. \& Chant, J. A localized GTPase exchange factor, Bud5, determines the orientation of division axes in yeast. Curr Biol 11, 803-807 (2001).

8. Zheng, Y., Bender, A. \& Cerione, R.A. Interactions among proteins involved in bud-site selection and bud-site assembly in Saccharomyces cerevisiae. J Biol Chem 270, 626-630 (1995).

9. Irazoqui, J.E., Gladfelter, A.S. \& Lew, D.J. Scaffold-mediated symmetry breaking by Cdc42p. Nat Cell Biol 5, 1062-1070 (2003).

10. Drubin, D.G. \& Nelson, W.J. Origins of cell polarity. Cell 84, 335-344 (1996).

11. Chant, J. \& Herskowitz, I. Genetic control of bud site selection in yeast by a set of gene products that constitute a morphogenetic pathway. Cell 65, 1203-1212 (1991).

12. Kang, P.J., Beven, L., Hariharan, S. \& Park, H.O. The Rsr1/Bud1 GTPase interacts with itself and the Cdc42 GTPase during bud-site selection and polarity establishment in budding yeast. Mol Biol Cell 21, 3007-3016 (2010).

13. Butty, A.C., Pryciak, P.M., Huang, L.S., Herskowitz, I. \& Peter, M. The role of Far1p in linking the heterotrimeric G protein to polarity establishment proteins during yeast mating. Science 282, 1511-1516 (1998).

14. Nern, A. \& Arkowitz, R.A. A Cdc24p-Farlp-Gbetagamma protein complex required for yeast orientation during mating. J Cell Biol 144, 1187-1202 (1999).

15. Nern, A. \& Arkowitz, R.A. A GTP-exchange factor required for cell orientation. Nature 391, 195-198 (1998).

16. Jackson, C.L. \& Hartwell, L.H. Courtship in S. cerevisiae: both cell types choose mating partners by responding to the strongest pheromone signal. Cell 63, 1039-1051 (1990).

17. Madden, K. \& Snyder, M. Specification of sites for polarized growth in Saccharomyces cerevisiae and the influence of external factors on site selection. Mol Biol Cell 3, 1025-1035 (1992).

18. Dorer, R., Pryciak, P.M. \& Hartwell, L.H. Saccharomyces cerevisiae cells execute a default pathway to select a mate in the absence of pheromone gradients. J Cell Biol 131, 845-861 (1995).

19. Segall, J.E. Polarization of yeast cells in spatial gradients of alpha mating factor. Proceedings of the National Academy of Sciences of the United States of America 90, 8332-8336 (1993).

20. Wang, X. et al. Mating yeast cells use an intrinsic polarity site to assemble a pheromone-gradient tracking machine. J Cell Biol 218, 3730-3752 (2019).

21. Abdul-Ganiyu, R. et al. Phosphorylated Gbeta is a directional cue during yeast gradient tracking. Sci Signal 14 (2021).

22. Park, H.O., Sanson, A. \& Herskowitz, I. Localization of Bud2p, a GTPase-activating protein necessary for programming cell polarity in yeast to the presumptive bud site. Genes Dev 13, 1912-1917 (1999).

23. Michelitch, M. \& Chant, J. A mechanism of Bud1p GTPase action suggested by mutational analysis and immunolocalization. Curr Biol 6, 446-454 (1996).

24. Cullen, P.J. \& Sprague, G.F., Jr. The regulation of filamentous growth in yeast. Genetics 190, 23-49 (2012).

25. Vasen, G., Dunayevich, P., Constantinou, A. \& Colman-Lerner, A. GPCR receptor phosphorylation and endocytosis are not necessary to switch polarized growth between internal cues during pheromone response in S. cerevisiae. Commun Integr Biol 13, 128-139 (2020).

26. Miller, K.E., Lo, W.C., Chou, C.S. \& Park, H.O. Temporal regulation of cell polarity via the interaction of the Ras GTPase Rsr1 and the scaffold protein Bem1. Mol Biol Cell 30, 2543-2557 (2019).

27. Liu, D. \& Novick, P. Bem 1 p contributes to secretory pathway polarization through a direct interaction with Exo70p. J Cell Biol 207, 59-72 (2014). 
28. Boyd, C., Hughes, T., Pypaert, M. \& Novick, P. Vesicles carry most exocyst subunits to exocytic sites marked by the remaining two subunits, Sec3p and Exo70p. J Cell Biol 167, 889-901 (2004).

29. Liu, D., Li, X., Shen, D. \& Novick, P. Two subunits of the exocyst, Sec3p and Exo70p, can function exclusively on the plasma membrane. Mol Biol Cell 29, 736-750 (2018).

30. Hutagalung, A.H., Coleman, J., Pypaert, M. \& Novick, P.J. An internal domain of Exo70p is required for actinindependent localization and mediates assembly of specific exocyst components. Mol Biol Cell 20, 153-163 (2009).

31. Guo, W., Tamanoi, F. \& Novick, P. Spatial regulation of the exocyst complex by Rho1 GTPase. Nat Cell Biol 3, 353-360 (2001).

32. Dyer, J.M. et al. Tracking shallow chemical gradients by actin-driven wandering of the polarization site. Curr Biol 23, 32-41 (2013).

33. McClure, A.W. et al. Role of Polarized G Protein Signaling in Tracking Pheromone Gradients. Dev Cell 35, 471-482 (2015).

34. Johnston, G.C., Prendergast, J.A. \& Singer, R.A. The Saccharomyces cerevisiae MYO2 gene encodes an essential myosin for vectorial transport of vesicles. J Cell Biol 113, 539-551 (1991).

35. Drubin, D.G., Miller, K.G. \& Botstein, D. Yeast actin-binding proteins: evidence for a role in morphogenesis. $J$ Cell Biol 107, 2551-2561 (1988).

36. Asakura, T. et al. Isolation and characterization of a novel actin filament-binding protein from Saccharomyces cerevisiae. Oncogene 16, 121-130 (1998).

37. Schott, D., Ho, J., Pruyne, D. \& Bretscher, A. The COOH-terminal domain of Myo2p, a yeast myosin V, has a direct role in secretory vesicle targeting. J Cell Biol 147, 791-808 (1999).

38. Gimeno, C.J., Ljungdahl, P.O., Styles, C.A. \& Fink, G.R. Unipolar cell divisions in the yeast S. cerevisiae lead to filamentous growth: regulation by starvation and RAS. Cell 68, 1077-1090 (1992).

39. Bi, E. \& Park, H.O. Cell polarization and cytokinesis in budding yeast. Genetics 191, 347-387 (2012).

40. Roberts, R.L. \& Fink, G.R. Elements of a single MAP kinase cascade in Saccharomyces cerevisiae mediate two developmental programs in the same cell type: mating and invasive growth. Genes Dev 8, 2974-2985 (1994).

41. Motegi, F., Plachta, N. \& Viasnoff, V. Novel approaches to link apicobasal polarity to cell fate specification. Curr Opin Cell Biol 62, 78-85 (2020).

42. Zhu, M. \& Zernicka-Goetz, M. Building an apical domain in the early mouse embryo: Lessons, challenges and perspectives. Curr Opin Cell Biol 62, 144-149 (2020).

43. Naganathan, S.R. et al. Morphogenetic degeneracies in the actomyosin cortex. Elife 7 (2018).

44. Zenker, J. et al. Expanding Actin Rings Zipper the Mouse Embryo for Blastocyst Formation. Cell 173, 776-791 e717 (2018).

45. Taniguchi, K. et al. An apicosome initiates self-organizing morphogenesis of human pluripotent stem cells. $J$ Cell Biol 216, 3981-3990 (2017).

46. Ausubel, F.M. et al. Current Protocols in Molecular Biology. (John Wiley and Sons, Inc., 1994).

47. Guthrie, C. \& Fink, G.R. Guide to Yeast Genetics and Molecular Biology., Vol. 351. (Academic Press, San Diego, CA.; 2002).

48. Sherman, F., G. R. Fink, J. B. Hicks, Eds., Laboratory Course Manual For Methods in Yeast Genetics. ((Cold Spring Harbor Laboratory Press, Cold Spring Harbor, New York, 1986)).

49. Reed, S.I., Hadwiger, J.A. \& Lorincz, A.T. Protein kinase activity associated with the product of the yeast cell division cycle gene CDC28. Proceedings of the National Academy of Science USA 82, 4055-4059 (1985). 